Research Paper

\title{
The MUC1 Ectodomain: A Novel and Eificient Target for Gold Nanoparticle Clustering and Vapor Nanobubble Generation
}

\author{
Brian P. Danysh ${ }^{1, *}$, Pamela E. Constantinou1, ${ }^{1,}$, Ekaterina Y. Lukianova-Hleb1,, \\ D. Carson ${ }^{1,3, 凶}$
}

1. Department of Biochemistry and Cell Biology, Rice University, Houston, TX 77005, USA;

2. Department of Physics and Astronomy, Rice University, Houston, TX 77005, USA;

3. Department of Biochemistry and Molecular Biology, MD Anderson Cancer Center, Houston, TX 77030, USA.

* Contributed equally to this work.

Corresponding author: dcarson@rice.edu

( ) Ivyspring International Publisher. This is an open-access article distributed under the terms of the Creative Commons License (http://creativecommons.org/ licenses/by-nc-nd/3.0/). Reproduction is permitted for personal, noncommercial use, provided that the article is in whole, unmodified, and properly cited.

Received: 2012.04.19; Accepted: 2012.05.21; Published: 2012.08.09

\begin{abstract}
$\mathrm{MUCl}$ is a large, heavily glycosylated transmembrane glycoprotein that is proposed to create a protective microenvironment in many adenocarcinomas. Here we compare $\mathrm{MUCl}$ and the well studied cell surface receptor target, EGFR, as gold nanoparticle (AuNP) targets and their subsequent vapor nanobubble generation efficacy in the human epithelial cell line, HES. Although EGFR and MUCI were both highly expressed in these cells, TEM and confocal images revealed $\mathrm{MUCl}$ as a superior target for nanoparticle intracellular accumulation and clustering. The MUCl-targeted AuNP intracellular clusters also generated significantly larger vapor nanobubbles. Our results demonstrate the promising opportunities $\mathrm{MUCl}$ offers to improve the efficacy of targeted nanoparticle based approaches.
\end{abstract}

Key words: MUC1, EGFR, Targeted Gold Nanoparticle, Vapor Nanobubble, Nanoparticle Endocytosis, Nanoparticle Clustering.

\section{Introduction}

Transmembrane mucin glycoproteins perform important barrier functions in mucosal epithelia. Their large size, heavy O- and N-linked glycosylation, and concentration at the apical cell surface make these molecules highly effective in this aspect of normal cell physiology. MUC1 is a well-studied mucin expressed by epithelial tissues of the stomach, pancreas, lung, trachea, kidney, salivary and mammary glands, and the female reproductive tract (1-3). MUC1's barrier functionality is exploited by many carcinomas as it is both overexpressed and distributed over the entire cell surface creating a local microenvironment, which protects these depolarized cancer cells from the host immune system and promotes metastatic activity (4-8). The ectodomain of MUC1 extends 200-500 nm from the cell surface and contains a tandem repeat motif of 20 amino acids rich in serine, threonine, and proline $(9,10)$. Moreover, the steric and charged properties of this mucin barrier inhibits the uptake of many hydrophobic chemotherapeutic drugs $(8,11$, $12)$, preferentially protecting cells within a tumor expressing high levels of MUC1 from antitumor treatments.

Due to its large size, accessibility, and abundant expression in many adenocarcinomas, MUC1 has been investigated as a potential target for directed 
therapies (reviewed in (13)). Antibodies recognizing the tandem repeat sequence in the large ectodomain have been conjugated to isotopes and drugs for targeted delivery (14-16). More recently, MUC1 has been used as a target for nanotherapies. Quantum dots conjugated to an aptamer recognizing MUC1 selectively accumulated in tumors in mice versus their non-targeted counterparts (17). In another study, AuNPs labeled with a MUC1 antibody, PAM4, were exposed to radiofrequency radiation in a pancreatic cancer mouse model. This allowed for cancer cells, which had a higher uptake of the AuNPs than the healthy cells, to be destroyed upon irradiation (18). MUC1 has also been used in a combination targeting approach where a single domain antibody to MUC1 was conjugated to a polymer nanocarrier containing a lethal transgene regulated by a MUC1 promoter in vitro (19). A MUC1 aptamer conjugated to paclitaxel loaded polymer nanoparticles showed higher uptake of the drug to MUC1 expressing cultured cells (20). Unlike other targeted nanotherapies, which rely on the targeting agent to remain intact through delivery via the vasculature system, MUC1 is found in the epithelial cells and, in many cases, localized, topical delivery can be implemented to facilitate efficiency of the targeting agent. In addition, the large size and accessibility at the cell surface, coupled with the occurrence of tandem repeat regions yields the potential of a single MUC1 molecule to bind multiple nanoparticles, amplifying the targeting signal.

The use of AuNPs for cancer therapies has become widespread in recent years due to their diagnostic and therapeutic potential determined by size, remarkable optical properties, low toxicity, and surface chemistry which allows them to be readily modified with targeting agents (antibodies, aptamers) to direct their delivery. AuNPs have been used as carriers for contrast agents, drugs and siRNAs (21, 22). AuNPs have the ability to scatter and absorb visible and near infrared light and have been investigated as both imaging $(23,24)$ and therapeutic tools $(25)$ in biomedical applications. However, background scattering by cells and tissues often interferes and can result in low sensitivity. The photothermal effects of AuNPs have been utilized in therapeutic techniques such as hyperthermia $(26,27)$; however, these treatments take up to minutes to achieve results and can damage adjacent normal tissues.

Employing the photothermal properties of gold, combined with MUC1 and similar mucins may improve the localized efficacy of MUC1-based diagnostics and therapeutics. Here we show that AuNPs conjugated to a MUC1 antibody recognizing the tandem repeat regions in the ectodomain are efficiently and specifically delivered to MUC1 expressing cells where they form large intracellular clusters. These clusters are detected with the photothermal method based upon short-pulsed laser excitation of AuNPs and subsequent generation of transient vapor nanobubbles (28-31). This approach allows detection of AuNPs in single cells with high sensitivity. We have compared our MUC1 targeted results with another well studied cell surface receptor target, EGFR, and have found that MUC1 is a superior target for AuNP targeting in the human epithelial cell line, HES.

\section{Methods}

\section{Cell culture}

HES cells were kindly provided by Dr. Doug Kniss (Ohio State University, Columbus, $\mathrm{OH}$ ) and HS-5 (CRL-11882) cells were obtained from American Type Culture Collection (atcc.org, Manassas, VA). HES and HS- 5 cells were maintained at $37^{\circ} \mathrm{C}$ in an atmosphere of air/ $\mathrm{CO}_{2}[95: 5(\mathrm{v} / \mathrm{v})]$. HES cells were supplemented in high glucose DMEM (Gibco Life Technologies, Grand Island, NY), supplemented with $5 \%(\mathrm{v} / \mathrm{v})$ fetal bovine serum (FBS; Atlanta Biologicals, Lawrenceville, GA), and $1 \mathrm{mM}$ sodium pyruvate (Sigma, St. Louis, MO). HS-5 cells were maintained in low glucose DMEM (Gibco Life Technologies, Grand Island, NY), supplemented with $10 \%(\mathrm{v} / \mathrm{v})$ FBS (Atlanta Biologicals, Lawrenceville, GA). For experiments, cells were grown as individual or co-cultures on removable 2-well Lab-Tek ${ }^{\mathrm{TM}}$ II Chambered Slides $^{\mathrm{TM}}$ (Nunc, Rochester, NY).

\section{Western Blots}

Cell lysates were solubilized in sample extraction buffer: $8 \mathrm{M}$ urea; $1 \%(\mathrm{w} / \mathrm{v})$ SDS; $50 \mathrm{mM}$ Tris, $\mathrm{pH}$ 7.0; $1 \%(\mathrm{v} / \mathrm{v}) \beta$-mercaptoethanol; and a 1:100 dilution of protease inhibitor cocktail (Sigma, St. Louis, MO). Protein extracts were incubated for $5 \mathrm{~min}$ at $100^{\circ} \mathrm{C}$ with Laemmli sample buffer (32) and separated by SDS-PAGE using a $10 \%(\mathrm{w} / \mathrm{v})$ Porzio and Pearson SDS-PAGE gel (33). Proteins were transferred from gels to Trans Blot Transfer Medium nitrocellulose membranes (Bio-Rad Laboratories, Hercules, CA) at $4^{\circ} \mathrm{C}$ for $5 \mathrm{~h}$ at $40 \mathrm{~V}$. Blots were blocked at room temperature for $6-8 \mathrm{~h}$ in PBS plus $0.1 \%(\mathrm{v} / \mathrm{v})$ Tween-20 (PBS-T) and $3 \%(\mathrm{w} / \mathrm{v})$ bovine serum albumin (BSA). Blots were probed overnight at $4^{\circ} \mathrm{C}$ (constant rotary agitation) with primary antibodies specific for: MUC1 ectodomain (214D4, kindly provided as hybridoma media by Dr. John Hilkens, The Netherlands Cancer Institute, Amsterdam, The Netherlands (34)) at a dilution of 1:1,000; a rabbit polyclonal antibody that recognizes all cell-assoiciated MUC1 (35), CT-1 (at a 
dilution of 1:2,500; EGFR mouse monoclonal antibody clone H9B4 (Invitrogen Life Technologies, Grand Island, NY) at a dilution of 1:5,000, and $\beta$-actin mouse monoclonal antibody clone 8226 (Abcam, Cambridge, MA) at a dilution of 1:10,000. Blots were rinsed (3 $\times 5$ min) at room temperature with PBS-T. Subsequently, blots were incubated for $2 \mathrm{~h}$ at $4^{\circ} \mathrm{C}$ with peroxidase conjugated a secondary antibody, either sheep anti-mouse or goat anti-rabbit (Jackson Immunoresearch, West Grove, PA) at final dilutions of $1: 100,00$ and 1:200,000 respectively, in $3 \%(\mathrm{w} / \mathrm{v})$ BSA/PBS-T. Finally, the blots were rinsed three times with PBS for $(3 \times 5 \mathrm{~min})$ at room temperature, and antibody binding was detected using the SuperSignal West Dura Extended Duration Substrate ECL system (Pierce, Rockford, IL) as described by the manufacturer. Blots then were exposed to x-ray film and analyzed by densitometry.

\section{Preparation of AuNPs}

Gold colloidal nanoparticles (AuNPs; $60 \mathrm{~nm}$ ) and antibody conjugation were prepared commercially (BioAssay Works, Ijamsville, MD) at concentrations of $50 \mathrm{OD}$. AuNPs were sterile filtered $(0.22 \mu \mathrm{m})$ and stored in 0.1X PBS and 0.1\% (w/v) BSA for up to 3 months. The antibodies, 214D4 (Millipore) and C225 (Cetuximab), were conjugated directly to the surface of the AuNPs, taking advantage of dative bonding between cysteine thiol groups of the antibody and the gold surface. An approximate ratio of 340 to 1 (antibody to AuNP) was used in the highly reproducible conjugation reaction and resulted in antibody-AuNP conjugates with an average hydrodynamic radius of the $84.6 \mathrm{~nm}(+/-5.5 \mathrm{~nm})$, as measured by dynamic light scattering. Prior to incubation with cells, AuNPs were diluted in phenol red free media, and their concentration adjusted at $\mathrm{A}_{548}$ to $0.22 \mathrm{OD}$. In experiments where both 214D4-AuNPs and C225-AuNPs were used in simultaneous incubations, the final concentration at $\mathrm{A}_{548}$ was approximately $0.44 \mathrm{OD}$.

\section{AuNP Incubation}

After reaching 50-70\% confluency, cells were washed three times with serum free DMEM to remove cell debris and soluble MUC1. Cells then were incubated with targeted AuNPs at $37^{\circ} \mathrm{C}$ on an orbital rocker for either 1 or 12 hours. Following incubation, cells were again washed three times with serum free, phenol red free DMEM (Gibco Life Technologies, Grand Island, NY) to remove unbound AuNPs. Slide chambers were removed and cells were covered with a \#1.5 coverslip and sealed.

\section{Transmission Electron Microscopy}

Cells were grown on Permanox ${ }^{\circledR}$ petri dishes and fixed with $2 \%(\mathrm{w} / \mathrm{v})$ glutaraldehyde and $2 \%(\mathrm{w} / \mathrm{v})$ paraformaldehyde in $0.1 \mathrm{M}$ sodium cacodylate buffer $(\mathrm{pH} 7.4)$ for $1 \mathrm{~h}$ at room temperature, then washed in $0.1 \mathrm{M}$ sodium cacodylate buffer ( $\mathrm{pH}$ 7.4). Cells were placed into fresh $2 \%(\mathrm{w} / \mathrm{v})$ glutaraldehyde and $2 \%$ $(\mathrm{w} / \mathrm{v})$ paraformaldehyde in $0.1 \mathrm{M}$ sodium cacodylate buffer $(\mathrm{pH} 7.4)$ and stored at $4^{\circ} \mathrm{C}$. Samples were washed with $0.1 \mathrm{M}$ sodium cacodylate buffer ( $\mathrm{pH} 7.4$ ) and then postfixed in $1 \%(\mathrm{w} / \mathrm{v})$ osmium tetroxide in buffer for $2 \mathrm{~h}$. Cells were washed, dehydrated in an ascending acetone series, gradually infiltrated with Embed-812 resin (Electron Microscopy Sciences, emsdiasum.com) and then polymerized at $60^{\circ} \mathrm{C}$ for 48 h. Ultrathin sections were cut on a Reichert-Jung UltracutE ultramicrotome and collected onto 200 mesh formvar-carbon coated copper grids. Sections were post-stained with saturated methanolic uranyl acetate and Reynolds' lead citrate and imaged at Delaware Biotechnology Institute (Newark, DE) on a Zeiss Libra 120 transmission electron microscope operated at 120 $\mathrm{kV}$. Digital images were captured with a Gatan Ultrascan $10002 \mathrm{k} \times 2 \mathrm{k}$ CCD camera. The diameters of the AuNP clusters in TEM images were determined using the line tool in ImageJ (imagej.nih.gov, v1.45i). Scale was set using the 200 or $500 \mathrm{~nm}$ scale bar in each image, then the diameter between the two furthest points along the perimeter of the particle cluster was measured. Two-tailed Student T-test used for statistical analysis.

\section{Confocal Microscopy}

Clustered AuNPs in targeted and non-targeted cells were evaluated using confocal microscopy (Zeiss, LSM710) Z-stack images in optical scattering mode and a $63 x$ oil immersion objective. For quantitative measurements, optical slice images $(1 \mu \mathrm{m}$ thick and taken at 2.5, 5.0, and $7.5 \mu \mathrm{m}$ from the apical cell surface) were analyzed using ImageJ (imagej.nih.gov, v1.45i). Dimensionality was reduced to remove channels used for cell location and orientation. Background was subtracted using a rolling ball radius of 50 for each image and the lower threshold was set to $\sim 3000$, clipping less intense pixels from the analysis. Maximum pixel values for each cluster were then measured using the analyze particle tool (particles were defined by at least 2 pixels). The pixel amplitude of reflected scattered light from the AuNP clusters correlates to its size (36). Two-level nested analysis of variance (ANOVA) was used for statistical analysis. 


\section{Generation and detection of vapor nanobub- bles around AuNP clusters}

To image and quantify the uptake of AuNPs by cells, we used the vapor bubbles generated around the clusters of AuNPs. Recently we demonstrated that vapor nanobubbles are triggered by the short-pulsed optical heating of AuNPs and has a threshold of generation that is sensitive to multiple variables including clustering of AuNPs (28-31, 37-39). Optical generation and detection of vapor nanobubbles were performed using a photothermal laser microscope (40,41). A 532 nm laser (STH-01, Standa Ltd, Vilnius, Lithuania) was used to irradiate single cells with a pulse duration of $500 \mathrm{ps}$ and a beam diameter of $15 \mu \mathrm{m}$. A laser pulse fluence was experimentally determined for each experiment to exceed the vapor nanobubble generation threshold in target cells (HES) and to be below the generation threshold for non-target cells. The fluence of each laser pulse was measured by registering its image and measuring of the beam diameter (at the level of 0.5 relative to the maximal intensity in the center of the beam) at the sample plane with the imaging device (Luka, Andor Technology, Belfast, Northern Ireland) and by measuring of the pulse energy using a pulse energy meter (Ophir Optronics, Ltd., Jerusalem, Israel). This scheme provided direct and precise measurements of the incident optical fluence at the cell plane for each excitation pulse. Custom software modules run on a PC and developed using the LabView platform was used to operate all hardware.

The superior optical scattering properties of vapor nanobubbles (28-31, 37-39) were used for their detection in individual cells with two probe laser beams, a pulsed probe beam $(576 \mathrm{~nm}, 70 \mathrm{ps}, 0.1$ $\left.\mathrm{mJ} / \mathrm{cm}^{2}\right)$ and a continuous probe laser $(633 \mathrm{~nm})$. This provided two independent signals: optical scattering time-resolved image pixel amplitude and duration of optical scattering time response (measured independently and simultaneously with the optical scattering image). Time-resolved optical scattering was used for imaging of the vapor nanobubbles while, the bubble specific time response was independently obtained from the continuous probe laser. The vapor nanobubble induced scattering of the probe beam decreased its axial amplitude, resulting in a dip-shaped output signal of the photodetector monitoring the probe beam. Thus, the time response of the probe laser radiation to the transient scattering effect of the vapor nanobubbles was registered. This mode provided the monitoring of bubble growth and collapse, delivering the bubble lifetime that characterizes its maximal diameter $(30,37,38,40,42-44)$. The lifetime was measured as the duration of the bub- ble-specific signal at half of the maximum amplitude level of the vapor nanobubble response. The vapor nanobubble lifetimes for each population of target and non-target cells were obtained by averaging data obtained from individual cells (20-30) over five different comparative experiments. In addition, we measured the probability of vapor nanobubble generation at a specific fluence of the excitation laser pulse. This allowed us to determine the bubble generation threshold fluence as the excitation laser fluence that provides a vapor nanobubble generation probability of 0.5 .

\section{Results}

\section{Comparison of MUCI- and EGFR-targeted AuNPs in cells using three distinct methods}

\section{Confocal Microscopy}

We targeted 60-nm AuNPs to this ectodomain using a mouse monoclonal antibody (214D4) raised against the PDTR residues within the tandem repeats of MUC1's relatively large ectodomain. MUC1's constitutive endocytotic internalization $(45,46)$ allows for the formation of large 214D4-AuNPs clusters within endosomal vesicles, which are made visible using the reflected scattered light from a $633 \mathrm{~nm}$ source on confocal microscopy images. Following a one hour incubation in co-cultures containing MUC1 expressing (HES) and non-expressing (HS-5) human cell lines at $37^{\circ} \mathrm{C}$, the MUC1-directed particles demonstrated high specificity and accumulation in the targeted HES cells (Figure 1A, B), while low particle binding was observed in HS-5 cells. The potential for MUC1-targeted AuNPs to produce large intracellular clusters was compared to particles targeting the well-studied cell surface molecule, EGFR, using the monoclonal antibody C225. HES cells endogenously express both MUC1 and EGFR (Figure 1C). Confocal images were used to compare the size and distribution of clusters of both MUC1 and EGFR-targeted AuNPs throughout HES cells. The pixel intensity of the light reflected off AuNP clusters is proportional to the size of the cluster (36). MUC1-targeted AuNP clusters were 2.0, 2.3, and 2.7 times larger than the EGFR-targeted AuNP clusters at 2.5, 5.0, and 7.5 $\mu \mathrm{m}$, respectively from the apical cell surface (Figure 2A).

\section{Transmission Election Microscopy}

Analysis of transmission electron microscopic (TEM) images of HES cells incubated with either 214D4- or C225-AuNPs for 12 hours show more and larger internalized clusters of the MUC1-targeted particles (Figure 2A, B). More than twice as many 214D4-AuNP clusters, 39 compared to 18 , were ob- 
served in the analysis of an equal number of TEM sections. The MUC1-targeted particles also aggregated into significantly larger clusters than the EGFR-targeted particles, $268.7 \mathrm{~nm}$ and $89.6 \mathrm{~nm}$ diameters $(p<0.001)$, respectively (Figure $2 \mathrm{C}$ ). The largest 214D4-AuNP clusters were over $500 \mathrm{~nm}$ in diameter with $>25$ individual AuNPs (each $60 \mathrm{~nm}$ diameter) visible in the ultrathin sections $(70 \mathrm{~nm})$, whereas most of the C225-AuNPs consisted of one or two visible particles.

\section{Vapor Nanobubbles}

Vapor nanobubbles are rapidly expanding and contracting transient vapor bubbles generated following exposure to one or more, short laser pulse directed towards intracellular AuNP clusters. The imaging potential of these bubbles is correlated to their size, which is proportional to their lifetime and can be tuned based on laser pulse fluence and AuNP cluster size; larger vapor nanobubbles correlate to larger NP clusters and are more capable of mechanically disrupting the cell membrane (31). An example of this can be seen in HES cells incubated with 214D4-AuNPs for one hour. Irradiation with a single laser pulse of $72 \mathrm{~mJ} / \mathrm{cm}^{2}$ produced a small vapor nanobubble lasting $50 \mathrm{~ns}$ (Figure 3A-C), whereas, irradiation with $120 \mathrm{~mJ} / \mathrm{cm}^{2}$ generates vapor nanobubble lasting $250 \mathrm{~ns}$, (Figure 3D-F), large enough to visibly disrupt the cell membrane.

The specificity and large clusters formed by the
214D4-AuNPs make it an ideal vector for directed AuNP therapies. We compared the size and selectivity of vapor nanobubbles generated from MUC1- and EGFR-targeted AuNPs in HES (MUC1+/EGFR+) and HS-5 (MUC1-/EGFR+) cells. Both cell lines were incubated with equal concentrations of individual targeted particles or with a combination of both for one hour, and then individual cells were irradiated with a single laser pulse fluence of $42 \mathrm{~mJ} / \mathrm{cm}^{2}$. We observed comparatively smaller vapor nanobubbles in the non-targeted HS-5 cells incubated with either 214D4-AuNPs, C225-AuNPs, or a combination of both targeted particles. The vapor nanobubbles generated in HS- 5 cells had average bubble lifetimes of 17.5, 29.2, and $49.0 \mathrm{~ns}$, and restricted to a subset of cells, with bubble generation probabilities of $0.54,0.65$, and 0.65 , respectively (Figure 4A, B). In MUC1-expressing HES cells, the probability of generating a bubble in a cell was 1.0 for all treatments. The average bubble lifetime in the HES cells using the 214D4-targeted AuNPs was $150.8 \mathrm{~ns}, 73.7 \%$ larger than the $86.8 \mathrm{~ns}$ bubbles generated by C225-targeted AuNPs in the same cells $(p<0.01)$. Combining both MUC1 and EGFR-targeted AuNPs generated even larger vapor nanobubbles, lasting 196.3 ns. Vapor nanobubbles generated from the MUC1-targeted AuNPs were also more cell specific than those formed around the EGFR-targeted particles, with lifetime ratios (HES/HS-5) of 8.6 and 3.0 , respectively.
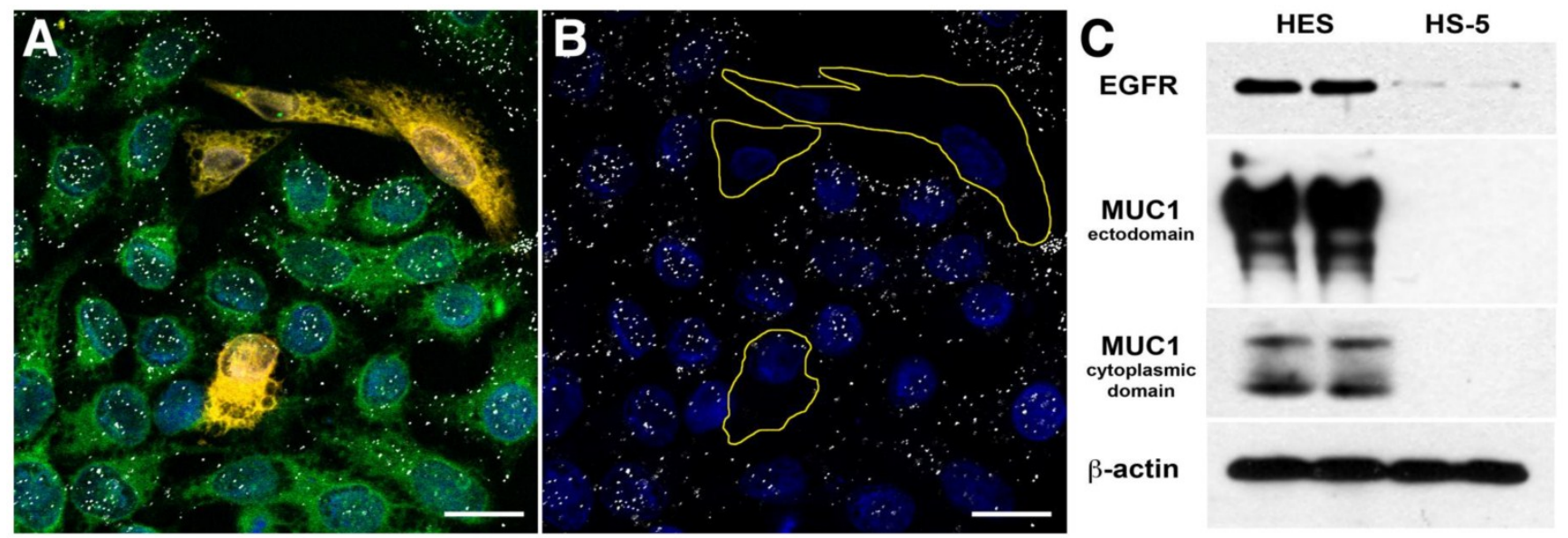

Figure I: Targeting Specificity of MUCI-targeted AuNPs. Confocal microscopy images of fluorescently labeled cells incubated with antibody-AuNP conjugates (white dots) in reflective mode. Reflected light from the clusters of MUCI targeted 2I4D4-AuNPs are visible in confocal microscope images as white dots on and within the HES cells but not the HS-5 cells. (A) Co-culture of HES (green) and HS-5 (orange) cells incubated with 2I4D4-AuNPs. (B) The fluorescent cell tracker channel is removed for clarity. (C) A western blot showing the relative $\mathrm{MUCI}(2$ I4D4 and CTI Abs) and EGFR expression in both cell lines. Scale bars $=20 \mu \mathrm{m}$. 

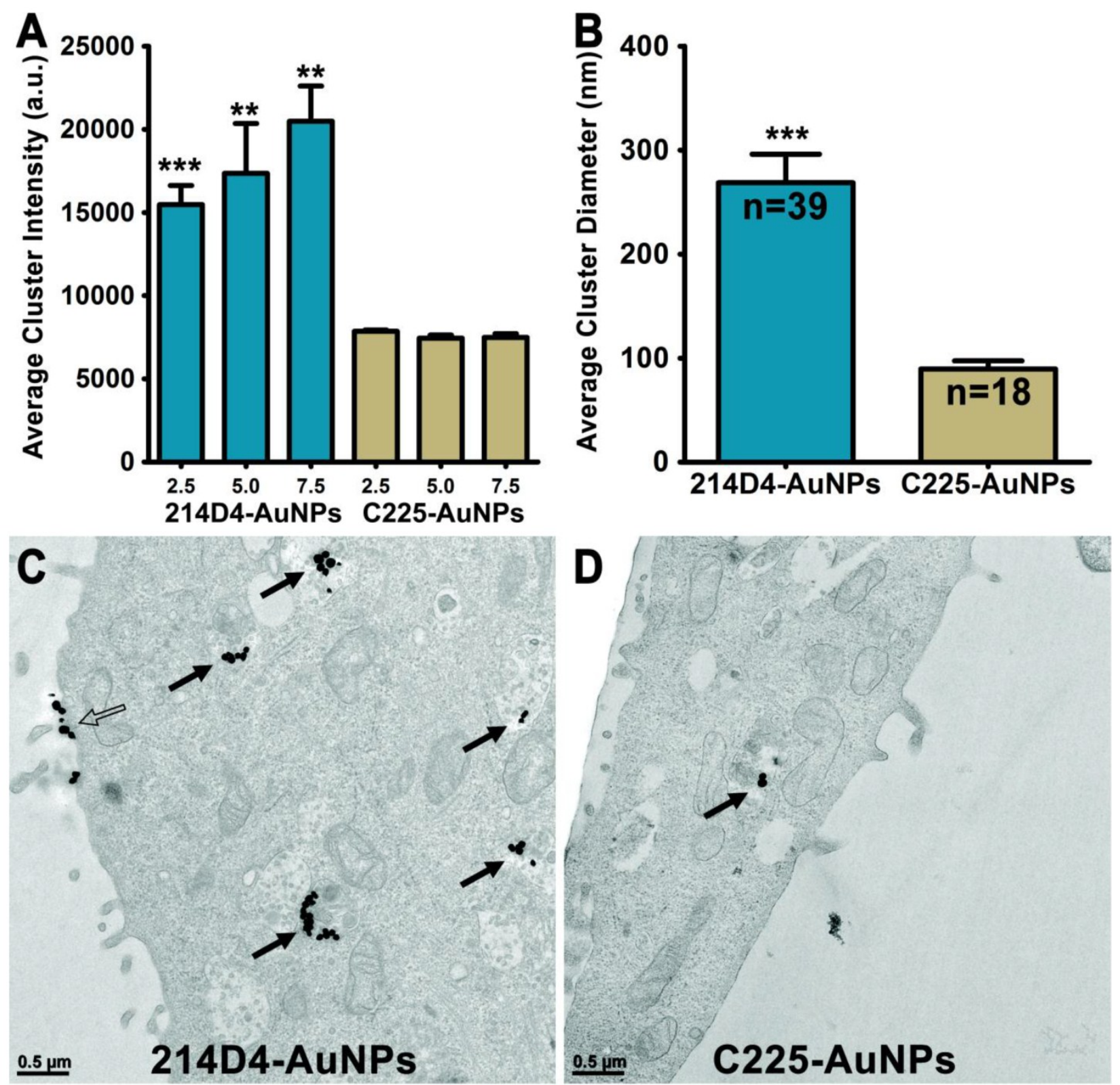

Figure 2: Endocytosis and Clustering of AuNPs. 214D4- and C225-AuNPs bind to either MUCI or EGFR on the cell surface and are endocytosed to form intracellular clusters. (A) Analysis of confocal microscopy images show 214D4-AuNPs form significantly larger clusters at three depths from the apical cell surface of HES cells, 2.5, 5.0, and $7.5 \mu \mathrm{m}$ (I $\mu \mathrm{m}$ optical slices), following a I-hour incubation with equal concentrations of targeted AuNPs. (B-D) TEM images depict intracellular (solid arrows) and extracellular (hollow arrow) AuNP clusters of various diameters in and around HES cells following a I2-hour incubation with equal concentrations of both targeted AuNPs. Each cluster consists of multiple $60 \mathrm{~nm}$ AuNPs (black dots). Clusters of MUCI-directed particles are significantly larger compared to EGFR-targeted particles (equal number of TEM sections analyzed). Error bars represent mean +/- SD. 


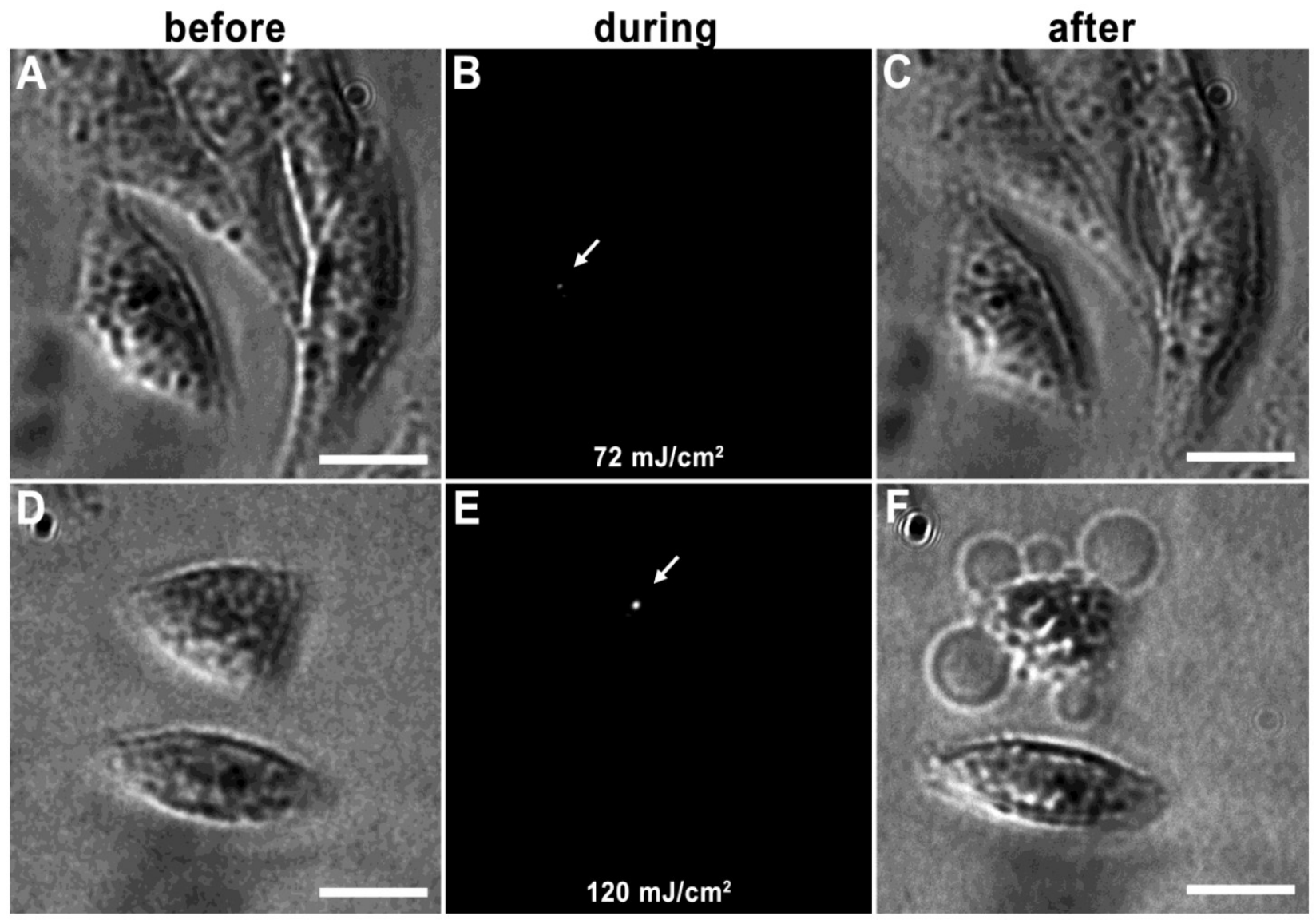

Figure 3: Tunable vapor nanobubbles. HES cells incubated with 214D4-AuNPs were exposed to a short laser pulse. The leftmost panels $(A$ and $D)$ depict cells after AuNP incubation prior to laser irradiation. The center panels (B and $E)$ show laser scattering off the vapor nanobubbles during their formation. The rightmost panels ( $C$ and $F$ ) show the effects of the vapor nanobubbles on the cells after laser irradiation. The higher laser pulse fluence $\left(120 \mathrm{~mJ} / \mathrm{cm}^{2}\right)$ generated a large bubble, which destroyed the cell membrane $(F)$. Scale bars $=10 \mu \mathrm{m}$.

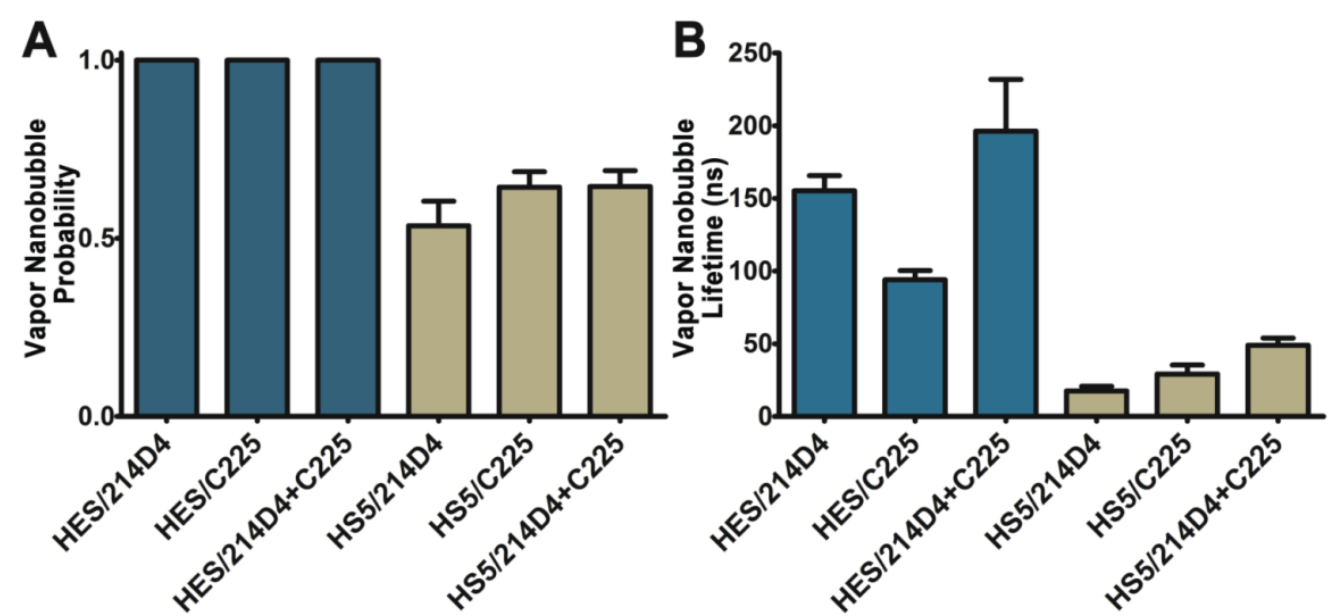

Figure 4: Comparison of vapor nanobubbles incidence and size. Vapor nanobubbles were generated from individual and combined treatments with MUCI-targeted (2I4D4) and EGFR-targeted (C225) AuNPs in both MUCI and EGFR positive HES cells (blue) and MUCI negative, EGFR positive HS-5 cells ( $\tan )$. Cells were incubated with AuNPs for I hour, and then individual cells were irradiated with a single laser pulse $\left(532 \mathrm{~nm}, 500 \mathrm{ps}, 72 \mathrm{~mJ} / \mathrm{cm}^{2}\right)$. A) The probability of producing a bubble in a cell following irradiation. B) The size of the transient vapor nanobubbles is proportional to their lifetimes. Error bars represent mean $+/-\mathrm{SD}$. 


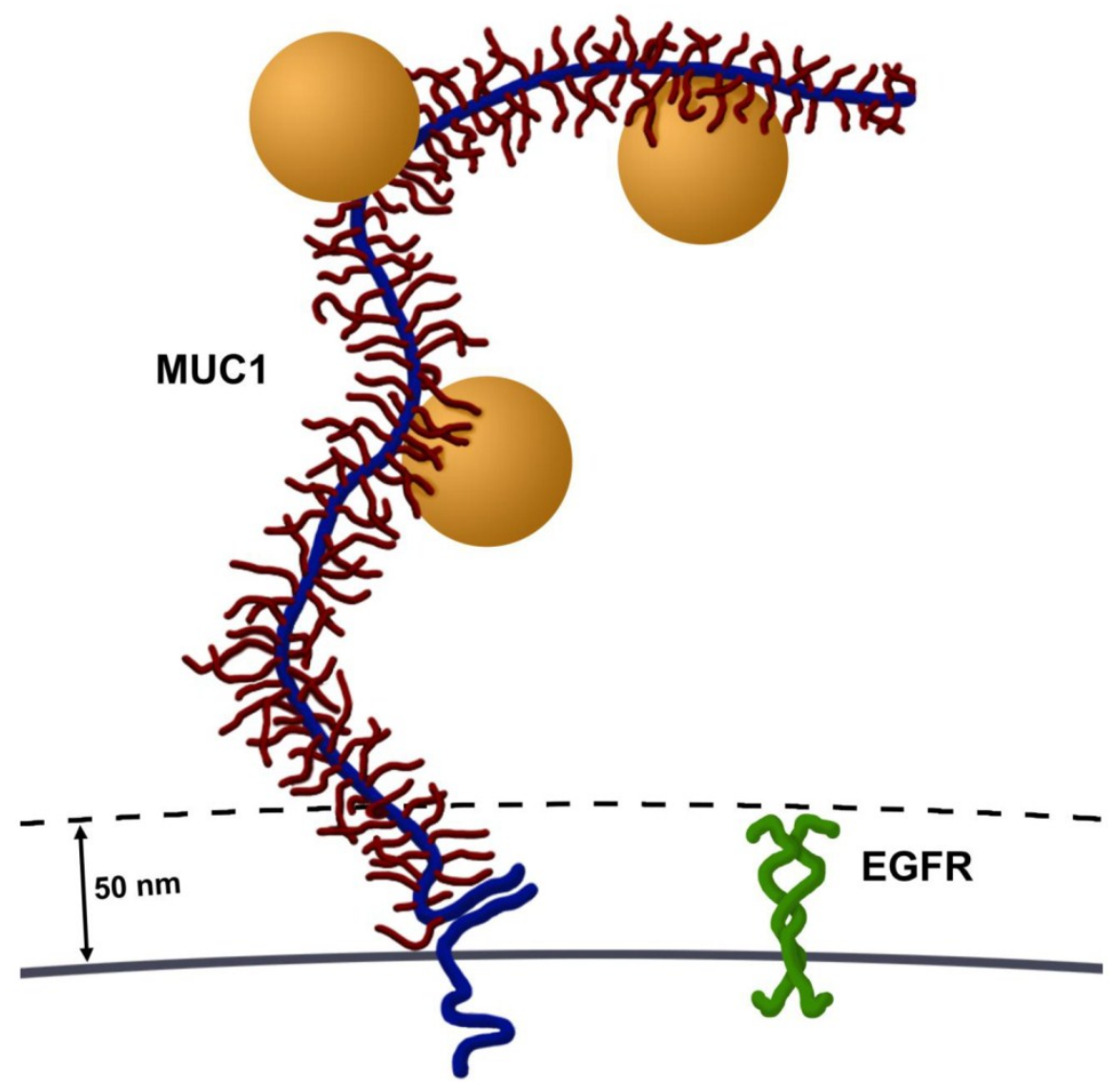

Figure 5: Size comparison of MUCI and EGFR. Most cell surface proteins, including EGFR homodimers (green), extend 30-50 nm from the cell surface. In contrast, MUCI's ectodomain (blue) is highly glycosylated (red) and rich in proline residues, giving it a highly extended structure, spanning 200-500 nm from the cell surface. Several AuNPs (orange spheres, $60 \mathrm{~nm}$ in diameter) are able to bind to tandem repeats in MUCl's ectodomain. Image drawn to scale.

\section{Discussion}

Mucins on the apical surface of normal mucosal epithelial cells serve both as a lubricant and a protective barrier. However in many cancer cells, MUC1 is overexpressed and the loss of cell polarity results in a distribution over the entire cell surface. The cell-surface receptor EGFR is also aberrantly over-expressed and distributed in many types of cancers (8) and has become a common target for nanoparticle-based cancer therapies (reviewed in (13)). The current study compares the use of MUC1- and EGFR-targeted AuNPs for intracellular clustering and vapor nanobubble-based diagnostics in a high MUC1 expressing human epithelial cell line. We demonstrated that the MUC1-targeted particles (214D4) specifically bind to MUC1 expressing cells and form larger clusters than the EGFR-targeted particles (C225 or cetuximab ${ }^{\circledR}$ ) using three distinct methods: optical scattering, electron microscopy, and vapor nanobubble sensing. Furthermore, we were able to effectively generate significantly larger vapor nanobubbles around the MUC1-targeted clusters than around the EGFR-targeted clusters.

There are two potential explanations for the larger particle clustering and vapor nanobubbles formed in these MUC1 and EGFR expressing cells (Figure 1C). First, it seems highly possible that targeting an epitope contained within the large number of tandem repeat motifs along MUC1's ectodomain potentially permits accumulation of multiple AuNPs on each molecule, compared to a single AuNP binding to EGFR. MUC1's ectodomain is rich in proline residues and in combination with a large number of O-linked oligosaccharides, give it a highly extended structure, spanning $200-500 \mathrm{~nm}$ from the cell surface (3). This potential accumulation of AuNPs per single MUC1 molecule permits cluster formation prior to endocytosis, which would result in larger aggregates. Second, MUC1's large extended (200-500 nm) ectodomain and hydrated sugars create a steric and hydrophilic barrier to diffusing molecules in normal epithelia. However, in contrast most cell surface proteins, including EGFR, extend only 30-50 nm from the 
cell surface (3) (Figure 5). Thus, over-expression of MUC1, as occurs in many adenocarcinomas, effectively obscures access of diffusing molecules to typical cell surface receptors, e.g., integrins and growth factor receptors such as EGFR. This same mechanism may account for the resistance of many adenocarcinoma cells that overexpress mucins to hydrophobic chemotherapeutic drugs (8). Although MUC1's ectodomain creates a formidable barrier, these same features make MUC1 an attractive target for nanoparticle based diagnostics and therapies.

Reducing or removing the barrier imposed by MUC1 should increase the efficacy of AuNPs targeting smaller cell surface receptors. MUC1 siRNA knockdown experiments were performed in HES cells and reduced MUC1 levels by $75-90 \%$; however, the effects on AuNP targeting were inconsistent. In most of the experiments endocytosis and clustering of 214D4-AuNPs significantly increased over controls (data not shown). We speculate the dense population of MUC1 in untreated HES cells sterically hinders access to its own ectodomain, similar to its proposed effect on EGFR. As MUC1 is knocked down, the barrier effects are reduced and 214D4-AuNPs gain access to the targeted epitope within the tandem repeat region. However, there appeared to be enough MUC1 still present to obstruct access to EGFR, as there was no significant difference in C225-AuNP clustering in the siRNA treated cells (data not shown). Since MUC1 co-localizes and binds with EGFR in various contexts in cancer cells $(47,48)$, it is also possible that this association compromises access to EGFR.

In the parameters of this study, MUC1 was shown to be a substantially better target than EGFR for AuNPs. However, EGFR is often used as a therapeutic target in many adenocarcinomas in which it is overexpressed. Glazer et al. compared both EGFR and MUC1 as candidates for AuNP mediated hyperthermic therapy using nonionizing radiofrequency radiation (18). These researchers found EGFR targeted (C225) AuNPs were more effective than MUC1 targeted (PAM4) particles at destroying pancreatic cancer xenografts in immune deficient mice. However, each targeting experiment was carried out on xenografts derived from different pancreatic cancer cell lines. EGFR-targeting experiments were performed on Panc-1 derived xenografts, which over express EGFR, but MUC1 is not detected (18); whereas, the MUC1-targeting experiments were performed on Capan-1 derived xenografts, which express both MUC1 and EGFR (18). Under these conditions, targeting inhibition of the smaller cell surface receptor would not be observed. Conversely, the study we present compares both AuNP targets in a single cell line (HES), allowing us to examine the targeting inhibition of smaller cell surface receptor like EGFR in the presence of MUC1.

An important factor determining diagnostic efficacy is the choice of targeting antibody. Ideally, a targeting molecule will have high specificity to epitopes present on cancer cells with minimal affinity to those present in normal tissues. The monoclonal antibody used by Glazer et al., PAM4, is highly specific to a MUC1 glycoform found in pancreatic adenocarcinoma and has little reactivity with pancreatitis and normal tissues $(49,50)$, with no reports of reactivity with other cancer types. Whereas, this study demonstrated the efficacy of a MUC1-targeted approach using a well-characterized monoclonal antibody (214D4) with specificity to a mature glycoform of MUC1 and high affinity towards HES cells. Many tumor cells aberrantly over express underglycosylated forms of MUC1, which expose new core protein and carbohydrate antigens (51). These antigens can be used to further distinguish normal MUC1 expressing mucosa from cancerous tissue with a catalog of available of glycol form specific antibodies (51). Moreover, since tumors are heterogeneous in terms of their expression of various molecules, including cell surface components, targeting MUC1 in combination with other cancer associated transmembrane proteins or other membrane bound mucins (MUC4 and MUC16) may prove to be an effective therapeutic approach.

MUC1's steric and hydrophilic properties create an obstacle for many therapeutic approaches $(1,2,8)$, especial when it is overexpressed. However, the nature of this molecule offers promising opportunities to improve not only the efficacy of the AuNP-based therapies and diagnostic approaches described, but also conventional drug and siRNA delivery therapies (13). Additionally, MUC1 is also the predominant mucin in uterine epithelia and is believed to prevent embryo implantation in humans during the non-receptive phase $(52,53)$. Use of MUC1 targeted AuNPs for local cell therapeutics and diagnostics may prove to be a useful tool to improve implantation success during in vitro fertilization procedures.

\section{Acknowledgements}

The work described was supported by Grant Number R01HD029963 to DDC and R01GM094816 to DOL from the National Institutes of Health; Grant Number P50CA098258 to DDC from the National Cancer Institute. Confocal microscopy was performed on equipment obtained through a Shared Instrumentation Grant Number S10RR02639901 from the National Institutes of Health. The content is solely the 
responsibility of the authors and does not necessarily represent the official views of the National Cancer Institute or the National Institutes of Health. We would also like to acknowledge Shannon Modla (Delaware Biotechnology Institute, Newark, DE) for her expert assistance with TEM microscopy.

\section{Conflict of Interests}

The authors have declared that no competing interest exists.

\section{References}

1. Hattrup CL, Gendler SJ. Structure and function of the cell surface (tethered) mucins. Annu Rev Physiol. 2008; 70: 431-57.

2. Gendler SJ. MUC1, the renaissance molecule. J Mammary Gland Biol Neoplasia. 2001; 6(3): 339-53.

3. Brayman M, Thathiah A, Carson DD. MUC1: a multifunctional cell surface component of reproductive tissue epithelia. Reprod Biol Endocrinol. 2004; 2: 4.

4. Komatsu M, Tatum L, Altman NH, Carraway CAC, Carraway KL. Potentiation of metastasis by cell surface sialomucin complex (rat MUC4), a multifunctional anti-adhesive glycoprotein. Int J Cancer. 2000; 87(4): 480-6.

5. Kondo K, Kohno N, Yokoyama A, Hiwada K. Decreased MUC1 expression induces E-cadherin-mediated cell adhesion of breast cancer cell lines. Cancer Res. 1998; 58(9): 2014-9.

6. Vandewielvankemenade E, Ligtenberg MJL, Deboer AJ, Buijs F, Vos HL, Melief CJM, et al. Episialin (Muc1) Inhibits Cytotoxic Lymphocyte-Target Cell-Interaction. J Immunol. 1993; 151(2): 767-76.

7. Wesseling J, Vandervalk SW, Vos HL, Sonnenberg A, Hilkens J. Episialin (Muc1) Overexpression Inhibits Integrin-Mediated Cell-Adhesion to Extracellular-Matrix Components. J Cell Biol. 1995; 129(1): 255-65.

8. Hollingsworth MA, Swanson BJ. Mucins in cancer: protection and control of the cell surface. Nat Rev Cancer. 2004; 4(1): 45-60.

9. Hilkens J, Vos HL, Wesseling J, Boer M, Storm J, van der Valk S, et al. Is episialin/MUC1 involved in breast cancer progression? Cancer Lett. 1995; 90(1): 27-33.

10. Bramwell ME, Wiseman G, Shotton DM. Electron-microscopic studies of the CA antigen, epitectin. J Cell Sci. 1986; 86: 249-61.

11. Satoh S, Hinoda Y, Hayashi T, Burdick MD, Imai K, Hollingsworth MA. Enhancement of metastatic properties of pancreatic cancer cells by MUC1 gene encoding an anti-adhesion molecule. Int J Cancer. 2000; 88(4): 507-18

12. Raina D, Ahmad R, Kumar S, Ren J, Yoshida K, Kharbanda S, et al. MUC1 oncoprotein blocks nuclear targeting of $\mathrm{c}-\mathrm{Abl}$ in the apoptotic response to DNA damage. EMBO J. 2006; 25(16): 3774-83.

13. Constantinou PE, Danysh BP, Dharmaraj N, Carson DD. Transmembrane mucins as novel therapeutic targets. Expert Rev Endocrinol Metab. 2011; 6(6): 835-48.

14. Verheijen RH, Massuger LF, Benigno BB, Epenetos AA, Lopes A, Soper JT, et al. Phase III trial of intraperitoneal therapy with yttrium-90-labeled HMFG1 murine monoclonal antibody in patients with epithelial ovarian cancer after a surgically defined complete remission. J Clin Oncol. 2006; 24(4): 571-8.

15. Oei AL, Massuger LF, Oyen WJ. Extraperitoneal leakage as a possible explanation for failure of one-time intraperitoneal treatment in ovarian cancer. Cancer Biother Radiopharm. 2007; 22(4): 508-14.

16. Rubinstein DB, Karmely M, Pichinuk E, Ziv R, Benhar I, Feng N, et al. The MUC1 oncoprotein as a functional target: immunotoxin binding to alpha/beta junction mediates cell killing. Int J Cancer. 2009; 124(1): 46-54.

17. Savla R, Taratula O, Garbuzenko O, Minko T. Tumor targeted quantum dot-mucin 1 aptamer-doxorubicin conjugate for imaging and treatment of cancer. J Control Release. 2011; 153(1): 16-22.

18. Glazer ES, Zhu C, Massey KL, Thompson CS, Kaluarachchi WD, Hamir $\mathrm{AN}$, et al. Noninvasive radiofrequency field destruction of pancreatic adenocarcinoma xenografts treated with targeted gold nanoparticles. Clin Cancer Res. 2010; 16(23): 5712-21.

19. Sadeqzadeh E, Rahbarizadeh F, Ahmadvand D, Rasaee MJ, Parhamifar L, Moghimi SM. Combined MUC1-specific nanobody-tagged PEG-polyethylenimine polyplex targeting and transcriptional targeting of tBid transgene for directed killing of MUC1 over-expressing tumour cells. J Control Release. 2011; 156(1): 85-91.
20. Yu C, Hu Y, Duan J, Yuan W, Wang C, Xu H, et al. Novel aptamer-nanoparticle bioconjugates enhances delivery of anticancer drug to MUC1-positive cancer cells in vitro. PLoS One. 2011; 6(9): e24077.

21. Ghosh P, Han G, De M, Kim CK, Rotello VM. Gold nanoparticles in delivery applications. Advanced Drug Delivery Reviews. 2008; 60(11): 1307-15.

22. Huang X, Jain PK, El-Sayed IH, El-Sayed MA. Gold nanoparticles: interesting optical properties and recent applications in cancer diagnostics and therapy. Nanomedicine (Lond). 2007; 2(5): 681-93.

23. El-Sayed IH, Huang X, El-Sayed MA. Surface plasmon resonance scattering and absorption of anti-EGFR antibody conjugated gold nanoparticles in cancer diagnostics: applications in oral cancer. Nano Lett. 2005; 5(5): 829-34.

24. Bickford LR, Agollah G, Drezek R, Yu TK. Silica-gold nanoshells as potential intraoperative molecular probes for HER2-overexpression in ex vivo breast tissue using near-infrared reflectance confocal microscopy. Breast Cancer Res Treat. 2010; 120(3): 547-55.

25. Loo C, Lowery A, Halas N, West J, Drezek R. Immunotargeted nanoshells for integrated cancer imaging and therapy. Nano Lett. 2005; 5(4): 709-11.

26. Hirsch LR, Stafford RJ, Bankson JA, Sershen SR, Rivera B, Price RE, et al. Nanoshell-mediated near-infrared thermal therapy of tumors under magnetic resonance guidance. Proc Natl Acad Sci U S A. 2003; 100(23): 13549-54

27. O'Neal DP, Hirsch LR, Halas NJ, Payne JD, West JL. Photo-thermal tumor ablation in mice using near infrared-absorbing nanoparticles. Cancer Lett. 2004; 209(2): 171-6.

28. Lapotko D. Pulsed photothermal heating of the media during bubble generation around gold nanoparticles. International Journal of Heat and Mass Transfer. 2009; 52(5-6): 1540-3.

29. Lapotko DO, Lukianova E, Oraevsky AA. Selective laser nano-thermolysis of human leukemia cells with microbubbles generated around clusters of gold nanoparticles. Lasers Surg Med. 2006; 38(6): 631-42.

30. Lukianova-Hleb E, Hu Y, Latterini L, Tarpani L, Lee S, Drezek RA, Hafner JH, Lapotko DO. Plasmonic nanobubbles as transient vapor nanobubbles generated around plasmonic nanoparticles. ACS Nano. 2010; 4(4): 2109-23.

31. Lukianova-Hleb EY, Hanna EY, Hafner JH, Lapotko DO. Tunable plasmonic nanobubbles for cell theranostics. Nanotechnology. 2010; 21(8): 85102.

32. Laemmli UK. Cleavage of structural proteins during the assembly of the head of bacteriophage T4. Nature. 1970; 227(5259): 680-5.

33. Porzio MA, Pearson AM. Improved resolution of myofibrillar proteins with sodium dodecyl sulfate-polyacrylamide gel electrophoresis. Biochim Biophys Acta. 1977; 490(1): 27-34.

34. Burchell J, Gendler S, Taylor-Papadimitriou J, Girling A, Lewis A, Millis $\mathrm{R}$, et al. Development and characterization of breast cancer reactive monoclonal antibodies directed to the core protein of the human milk mucin. Cancer Res. 1987; 47(20): 5476-82.

35. DeLoia JA, Krasnow JS, Brekosky J, Babaknia A, Julian J, Carson DD. Regional specialization of the cell membrane-associated, polymorphic mucin (MUC1) in human uterine epithelia. Hum Reprod. 1998; 13(1O): 2902-9.

36. Yguerabide J, Yguerabide EE. Light-scattering submicroscopic particles as highly fluorescent analogs and their use as tracer labels in clinical and biological applications - I. Theory. Anal Biochem. 1998; 262(2): 137-56.

37. Hleb EY, Lapotko DO. Photothermal properties of gold nanoparticles under exposure to high optical energies. Nanotechnology. 2008; 19(35).

38. Lukianova-Hleb E, Lapotko DO. Influence of transient environmental photothermal effects on optical scattering by gold nanoparticles. Nano Lett. 2009; 9(5): 2160-6.

39. Lukianova-Hleb EY, Ren X, Constantinou PE, Danysh BP, Shenefelt DL, Carson DD, et al. Improved Cellular Specificity of Plasmonic Nanobubbles versus Nanoparticles in Heterogeneous Cell Systems. PLoS One. 2012; 7(4): e34537.

40. Lapotko D. Optical excitation and detection of vapor bubbles around plasmonic nanoparticles. Opt Express. 2009; 17(4): 2538-56.

41. Lapotko D. Plasmonic nanoparticle-generated photothermal bubbles and their biomedical applications. Nanomedicine (Lond). 2009; 4(7): 813-45.

42. Neumann J, Brinkmann R. Nucleation dynamics around single microabsorbers in water heated by nanosecond laser irradiation. J Appl Phys. 2007; 101(11): 114701.

43. Vanleeuwen TG, Jansen ED, Motamedi M, Welch AJ, Borst C. Excimer-Laser Ablation of Soft-Tissue - a Study of the Content of 
Rapidly Expanding and Collapsing Bubbles. Ieee J Quantum Elect. 1994; 30(5): 1339-47.

44. Vogel A, Noack J, Huttman G, Paltauf G. Mechanisms of femtosecond laser nanosurgery of cells and tissues. Appl Phys B-Lasers O. 2005; 81(8): 1015-47.

45. Altschuler Y, Kinlough CL, Poland PA, Bruns JB, Apodaca G, Weisz OA, et al. Clathrin-mediated endocytosis of MUC1 is modulated by its glycosylation state. Molecular Biology of the Cell. 2000; 11(3): 819-31.

46. Liu X, Yuan Z, Chung M. MUC1 intra-cellular trafficking is clathrin, dynamin, and rab5 dependent. Biochem Biophys Res Commun. 2008; 376(4): 688-93.

47. Schroeder JA, Thompson MC, Gardner MM, Gendler SJ. Transgenic MUC1 interacts with epidermal growth factor receptor and correlates with mitogen-activated protein kinase activation in the mouse mammary gland. J Biol Chem. 2001; 276(16): 13057-64.

48. Bitler BG, Goverdhan A, Schroeder JA. MUC1 regulates nuclear localization and function of the epidermal growth factor receptor. J Cell Sci. 2010; 123(Pt 10): 1716-23.

49. Gold DV, Modrak DE, Ying Z, Cardillo TM, Sharkey RM, Goldenberg DM. New MUC1 serum immunoassay differentiates pancreatic cancer from pancreatitis. J Clin Oncol. 2006; 24(2): 252-8.

50. Gold DV, Karanjawala Z, Modrak DE, Goldenberg DM, Hruban RH. PAM4-reactive MUC1 is a biomarker for early pancreatic adenocarcinoma. Clin Cancer Res. 2007; 13(24): 7380-7.

51. van Leeuwen EB, Cloosen S, Senden-Gijsbers BL, Agervig Tarp M, Mandel U, Clausen $\mathrm{H}$, et al. Expression of aberrantly glycosylated tumor mucin-1 on human DC after transduction with a fiber-modified adenoviral vector. Cytotherapy. 2006; 8(1): 24-35.

52. Thathiah A, Carson DD. Mucins and blastocyst attachment. Rev Endocr Metab Disord. 2002; 3(2): 87-96.

53. Meseguer M, Aplin JD, Caballero-Campo P, O'Connor JE, Martin JC, Remohi J, et al. Human endometrial mucin MUC1 is up-regulated by progesterone and down-regulated in vitro by the human blastocyst. Biol Reprod. 2001; 64(2): 590-601. 